\title{
Study of Visco-Elastic Fluid Flow and Heat Transfer over a Stretching Sheet with Variable Viscosity and Thermal Radiation
}

\author{
M. Ramya ${ }^{1}$, K. Sangeetha ${ }^{2}$, M.Pavithra ${ }^{3}$ \\ ${ }^{1,2,3}$ Assistant Professors, Dr.Sns Rajalakshmi College Of Arts And Science,Coimbatore,India.
}

\begin{abstract}
This dissertation deals with the boundary layer flow and heat transfer in a visco-elastic fluid flow a stretching sheet in the presence of radiation. Two different temperature are studied here, (i) PST, that is the sheet with prescribed surface temperature (ii)PHF, that is the sheet with prescribed heat flux. The basic boundary layer equations for momentum and heat transfer which are non-linear parital differential equation is converted into non-linear ordinary differential equation using similarity transformation. The resulting nonlinear equation is solved using numerical shooting method for three unknown initial conditions with fourth order Runge-Kutta method. To know the physics of the problem, numerical results are discussed with the help of graphs for various of parameters, such as fluid viscosity parameter, visco-elastic parameter, Prandtl number, Eckert number, heat source/sink parameter and radiation parameter.
\end{abstract}

\section{Introduction}

Boundary layer behaviour over a moving continuous solid surface is an important type of flow occurring in general engineering processes. The heat transfer due to a continuously moving surface through an ambient fluid is one of the thrust areas of current research. Such investigations find their application over a broad spectrum of science and engineering disciplines respecially in the field of chemical engineering process like metallurgical process, polymer extrusion process involues cooling of a molten, liquid being stretched into a cooling system.

Sakiadis[19] presented the study of the boundary layer flow over a continuous solid surface moving with constant speed. Bataller[3] present a study of the flow and heat transfer of an

incompressible homogeneous second grade fluid over a non-isothermal stretching sheet. Using similarity transformation they convert the partial Differential equations to ordinary differential equations. Dual pal and Hiranmoy mondal[6] investigated the analysis of combined effects of Soret and Dufour on unsteady MHD nonDarcy mixed convection over a stretching sheet. Mohamed[11], investigated the effect of radiation on the heat and fluid flow over an unsteady stretching surface. Andersson[1] MHD flow of a visco elastic fluid past a stretching surface. Gupta[7] Heat and mass transfer in a stretching sheet suction or blowing. These coupled nonlinear equations, governing the problem, are reduced to a system of coupled non-linear higher-order ordinary differential equations by applting suitable similarity transformations. This resultant boundary value problem has been converted into the system of six-simultaneous equations of first order for six unknowns. Then this system is solved by employing a numerical shooting technique(for two unknown initial conditions) with fourth-order Runge-Kutta integration scheme. Computation is carried out for temperature and horizontal velocity profiles, Nusselt number and skin friction parameter when the walls are maintained with prescribed surface temperature and prescribed wall heat fluxes. Analyses have been made to investigate the effect of fluid viscosity, viscoelasticity, permeability of the porous medium and Prandtl number on the flow behavior and heat transfer process. Emphasis has been laid to study the effect of fluid viscosity on the other physical characteristics. One of the important findings in the present study is that the effect of fluid viscocity parameter is to decrease the temperature profile significantly when the flow is through a porous medium.

\section{Mathematical formulation}

Consider a steady laminar flow of an incompressible visco-elastic fluid (walter's liquid-B) in a porous medium past a semi-infinite stretching stretching sheet coinciding with a plane $\mathrm{y}=0$ and the $\mathrm{n}$ flow being continued to $y>0$, keeping the origin fixed, caused by the simultaneous application of two equal and opposite forces along the $\mathrm{x}$-axis which results in stretching of the sheet and hence the flow is generated due to the stretching of the sheet. We have $\mathrm{x}$-axis along the surface, $\mathrm{y}$-axis being normal to it and $\mathrm{u}$ and $\mathrm{v}$ are the fluid tangential velocity and normal velocity respectively.

$$
\frac{\partial u}{\partial x}+\frac{\partial v}{\partial y}=0
$$

$u \frac{\partial u}{\partial x}+v \frac{\partial T}{\partial y}=\frac{1}{\rho_{\infty}} \frac{\partial}{\partial y}\left(\mu \frac{\partial u}{\partial y}\right)-k_{0}\left\{u \frac{\partial^{3} u}{\partial x \partial y^{2}}+v \frac{\partial^{3} u}{\partial y^{3}}+\frac{\partial u \partial^{2} u}{\partial x \partial y^{2}}-\frac{\partial u \partial^{2} u}{\partial y \partial x \partial y}\right\}-\frac{v}{k^{\prime} u}$ 
$u \frac{\partial T}{\partial x}+v \frac{\partial T}{\partial y}=\frac{k}{\rho c_{p}} \frac{\partial^{2} T}{\partial y^{2}}+\frac{Q}{\rho c_{p}}\left(T-T_{\infty}\right)+\frac{\mu}{\rho c_{p}}\left(\frac{\partial u}{\partial y}\right)^{2}-\frac{1}{\rho c_{p}} \frac{\partial q_{r}}{\partial y}$

Where $k_{0}$ is the visco elastic parameter, $v$ is the kinematic viscosity, $k^{\prime}$ are permeability of the porous medium, $\mathrm{k}$ is the thermal conductivity of the fluid, $\mu$ is the coefficient of viscosity of the fluid, $T_{\infty}$ is the constant value of temperature and coefficient of viscosity far away from the sheet and qr is the radiative heat flux. The term Q represents the volumetric rate of heat generation. Eq. (2.2) has been derived with the assumption that the contribution from the normal stress is of the same order of magnitude as the shear stress, in addition to the usual boundary layer approximation. Here, $\mu$ is the coefficient of viscosity, which is considered to vary as a function of temperature as follows:

$\frac{1}{\mu}=\frac{1}{\mu_{\infty}}\left[1+\gamma\left(T-T_{\infty}\right)\right] \quad$ i.e $\frac{1}{\mu}=a\left(T-T_{r}\right)$

Where, $a=\frac{\gamma}{\mu_{\infty}} \quad$ and $\quad T_{r}=T_{\infty}-\frac{1}{\gamma}$

Are constants, $a>0$ corresponds to liquid and $a<0$ for gases. $T_{\infty}$ and $\mu_{\infty}$ are constant values of temperature and coefficient of viscosity, respectively, at far away from the sheet.

Here, we consider two more general cases of non-isothermal boundary conditions

(1) Surface with prescribed power-law temperature (PST)

(2) Surface with prescribed power-law heat flux (PHF)

The prescribed power-law surface temperature and power-law heat flux are considered to be functions of $\mathrm{x}$ only

The corresponding boundary conditions are $u=b x, v=0$

$T=T_{w}=T_{\infty}+A\left(\frac{x}{l}\right)$ at $y=0$, (PST Case)

$-k \frac{\partial T}{\partial y}=q_{w}=D\left(\frac{x}{l}\right)$ at $y=0$. (PHF Case)

$T \rightarrow T_{\infty}$ as $y \rightarrow \infty$,

Where $\mathrm{A}$ and $\mathrm{D}$ are constants, $T_{\infty}$ is the wall temperature of the sheet, $T_{\infty}$ is the constant values of temperature of the fluid far away from the sheet and 1 is the characteristic length. It is to be noted that Eq. (2.2) and (2.3) are of third order in $u$ and second order in T. The boundary conditions prescribed by Eq. (2.5) are sufficient to solve them. In order to obtain dimensionless form of the solution we define the following variables:

$u=b x f_{\eta}(\eta), v=-\left(b v_{\infty}\right)^{1 / 2} f(\eta), \eta=\left(\frac{b}{v_{\infty}}\right)^{1 / 2} y$,
$\left(T-T_{\infty}\right)=\left(T_{w}-T_{\infty}\right) \theta(\eta)$

Where

$$
\begin{array}{cc}
\left(T_{w}-T_{\infty}\right)=A\left(\frac{x}{l}\right) & \text { ( PST Case) } \\
=\frac{D}{k}\left(\frac{v_{\infty}}{b}\right)^{1 / 2}\left(\frac{x}{l}\right) & \text { (PHF Case) }
\end{array}
$$

(2.3) reduce to

Making use of these transformations, we notice that Eq. (2.1) is identically satisfied and Eq. (2.2) and

$$
\begin{aligned}
& \mathrm{f}_{\eta}{ }^{2}-\mathrm{ff}_{\eta \eta}=\frac{1}{1-\theta / \theta_{\mathrm{r}}}\left[\mathrm{f}_{\eta \eta \eta}-\frac{\mathrm{f}_{\eta \theta_{\eta}}}{\theta \theta_{\mathrm{r}}}\right]-\mathrm{k} 1\left[2 \mathrm{f}_{\eta} \mathrm{f}_{\eta \eta \eta}-\right. \\
& \theta_{\eta \eta}=\frac{R}{R+1}\left[\frac{-\operatorname{Pr} E}{1-\theta / \theta_{r}} \boldsymbol{f}_{\eta \eta}^{2}-\operatorname{Pr}\left(-\mathrm{f}_{\eta}+\beta\right) \theta-\operatorname{Prf} \theta_{\eta}\right]
\end{aligned}
$$$$
\left.\mathrm{ff}_{\eta \eta \eta \eta}-\mathrm{f}_{\eta \eta}^{2}\right]-\frac{\mathrm{k} 2}{1-{ }^{\theta} / \theta_{\mathrm{r}}} \mathrm{f}_{\eta}
$$

Boundary conditions of $\mathrm{Eq}(2.5)$ are transformed into

PST Case:

$$
\begin{array}{r}
f_{\eta}(\eta)=1, f(\eta)=0, \theta(\eta)=1 \text { at } \eta=0, \\
f_{\eta}(\eta)=0, f_{\eta \eta}(\eta)=0, \theta(\eta)=0 \text { as } \eta \rightarrow \infty
\end{array}
$$

$$
\begin{aligned}
f_{\eta}(\eta) & =1, \theta_{\eta}(\eta)=-1, f(\eta)=0 \text { at } \eta=0, \\
f_{\eta}(\eta)=0, f_{\eta \eta}(\eta)=0, \theta(\eta) & =0 \text { as } \eta \rightarrow \infty
\end{aligned}
$$

Here , $P_{r}=\frac{\mu C_{p}}{k}$ is the Prandtl number, $E=\frac{A}{\rho C_{p C^{2 l^{2}}}}$ is the Eckert number and $\theta_{r}=1 / v\left(T_{w}-T_{\infty}\right)$ is the viscosity number, $R=\frac{3 k^{*}}{16 \sigma^{* \mathrm{~T}^{3}} \infty}$ is the radiation parameter, $\beta=\frac{Q}{p C_{\rho b}}, E=\frac{b^{2} x l}{A}$ is the Eckert number.

Investigation on flow behavior and heat transfer would be carried out by analyzing the non-dimensional local shear stress $\tau_{w}$ (skin friction) and the rate of heat transfer coefficient $\mathrm{Nu}$ (Nusselt number) at the wall. These quantities are defined as

$$
\begin{aligned}
& \tau=\frac{\tau^{*}}{\Gamma b_{\mathrm{x}}} \sqrt{\frac{\mathrm{b}}{v_{\infty}}}=\mathrm{f}_{\eta \eta}(\eta) \text { at } \eta=0 \\
& \mathrm{Nu}=\frac{-\mathrm{h}}{\left(\mathrm{T}_{\mathrm{w}}-\mathrm{T}_{\infty}\right)} \mathrm{T}_{\mathrm{y}}=\theta_{\eta}(\eta) \text { at } \eta=0
\end{aligned}
$$




\section{Numerical solution}

The above equations (2.7) and (2.8) are nonlinear ordinary differential equation. Which constitute the nonlinear boundary value problem. As no prescribed method is available to solve nonlinear boundary value problem, it has to be reduced to an initial value problem. This process is done by Runge-Kutta shooting method. To initiate the shooting process. We have to make an initial guess judiciously for the values of $f^{\prime \prime \prime}(0), f^{\prime \prime}(0)$ and $\theta^{\prime}(0)$ (PHF case). The success of the procedure depends very much on how good the guess is .Numerical results are found for several values of the physical parameters $\mathrm{E}, \operatorname{Pr}, \mathrm{k} 1, \mathrm{k} 2, \theta_{r}, \mathrm{R}$.

\section{Results and discussion}

In the present work, I have analyzed the effect of fluid viscosity, visco-elasticity, permeability of the porous medium and prandtl number on the flow behavior and heat transfer process. The boundary layer partial differential equation which are highly non-linear, have been converted into set of ordinary differential equation by applying similarity transformation and they are solved by numerically using Runge-Kutta method.

Fig.1 Depicts the effect of radiation for various viscoelastic parameter on the heat transfer in the case of PST. Two plots reveal that the dimensionless temperature $\theta(\eta)$ versus $\eta$ from the wall, increases with increasing the values of visco-elastic parameter $(\mathrm{k} 1)$ and decreasing values of radiation parameter(R).Fig.2 Depicts the effect of radiation for various values of visco-elastic parameter on the heat transfer in the case of PST. Two plots reveal that the dimensionless velocity $f^{\prime}(\eta)$ versus $\eta$ from the wall, increases with increasing values of visco-elastic parameter $(\mathrm{k} 1)$ and decreasing values of radiation parameter $(\mathrm{R})$.

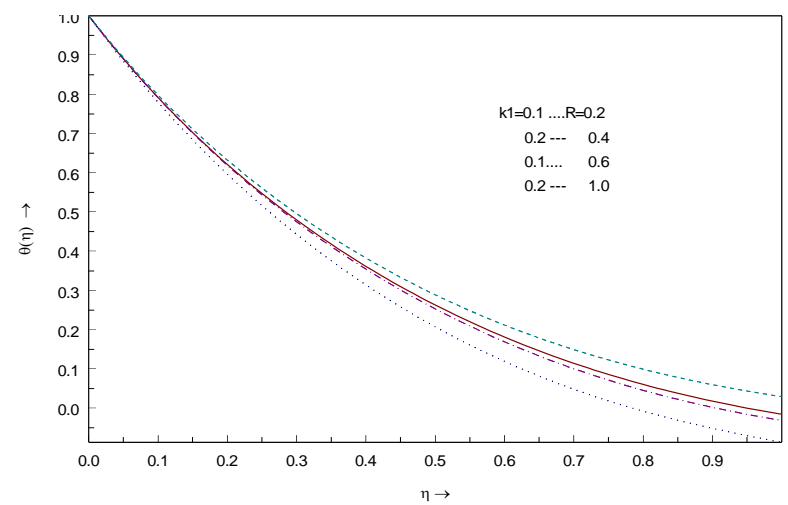

Fig.1 Effect of radiation parameter for various values of $\mathrm{K} 1$ in $\mathrm{PST}$ case with $\mathrm{Pr}=7.0, \mathrm{E}=0.02, \mathrm{~K} 2=0.0, \mathrm{~B}=-0.05$ for temperature profile

Fig 3. Depicts the effect of radiation for various of fluid viscosity parameter on the heat transfer in the case of PST. Two plots reveal that the dimensionless temperature $\theta(\eta)$ versus $\eta$ from the wall, increases with increasing values of fluid viscosity parameter (A) and decreasing values of radiation parameter (R).fig 4 . Demonstrates the effect of radiation for various values of prandtl number on the heat transfer in the case of PST. Two plots reveal that the dimensionless velocity $f^{\prime}(\eta)$ versus $\eta$ from the wall increases with increasing values of prandtl number $(\mathrm{Pr})$ and decreasing values of radiation parameter $(\mathrm{R})$. It is noticed from the figure that the effect of increasing values pf prandtl number and radiation parameter reduced the velocity profile in PST case.

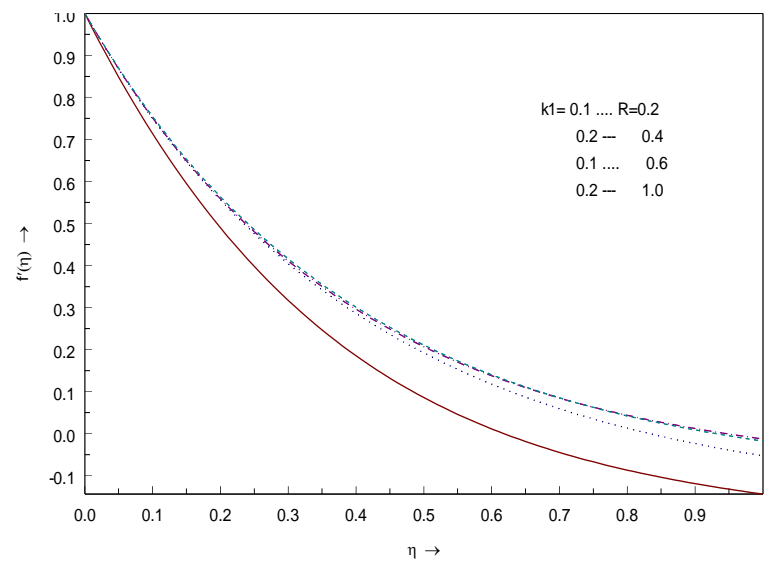

Fig.2 Effect of radiation parameter for various values of $\mathrm{K} 1$ in PST case with $\mathrm{Pr}=7.0, \mathrm{E}=0.02, \mathrm{~K} 2=1.0, \mathrm{~B}=-0.05$ for velocity profile. 
Fig 5. Illustrates the effect of radiation for various values of Eckert number on the heat transfer in the case of PST. Two plots reveal that the dimensionless temperature $\theta(\eta)$ versus $\eta$ from the wall increases with increasing values of Eckert number $(E)$ and decreasing values of radiation $(R)$. It is noticed from the figure that the effect of increasing values of Eckect number and radiation reduced the temperature profile in PST case.

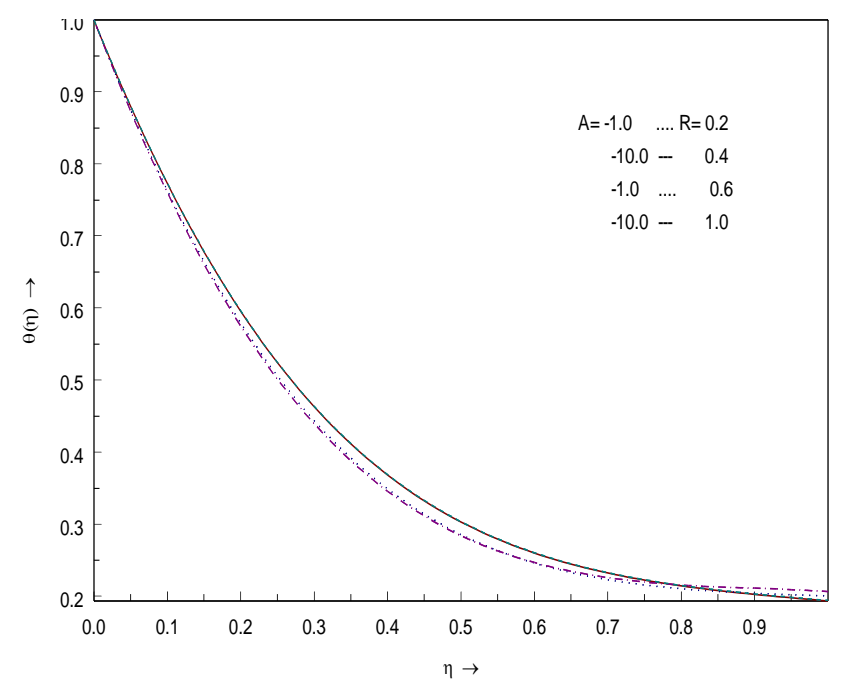

Fig.3 Effect of radiation parameter for various values of $\mathrm{A}$ in PST case with $\mathrm{Pr}=7.0, \mathrm{E}=0.02, \mathrm{~K} 1=0.1, \mathrm{~K} 2=0.2$, $\mathrm{B}=-0.05$ for temperature profile

Fig 6. Demonstrates the effect of radiation for various values of heat source/sink parameter on the heat transfer in the case of PST. Two plots reveal that the dimensionless velocity $f^{\prime}(\eta)$ versus $\eta$ from the wall, increases with increasing values of heat source/sink parameter $(\beta)$ and decreasing values of radiation parameter $(\mathrm{R})$. It is noticed from the figure that the effect of accelerate values of heat source/sink parameter and radiation parameter decelerate the velocity profile in PST case.

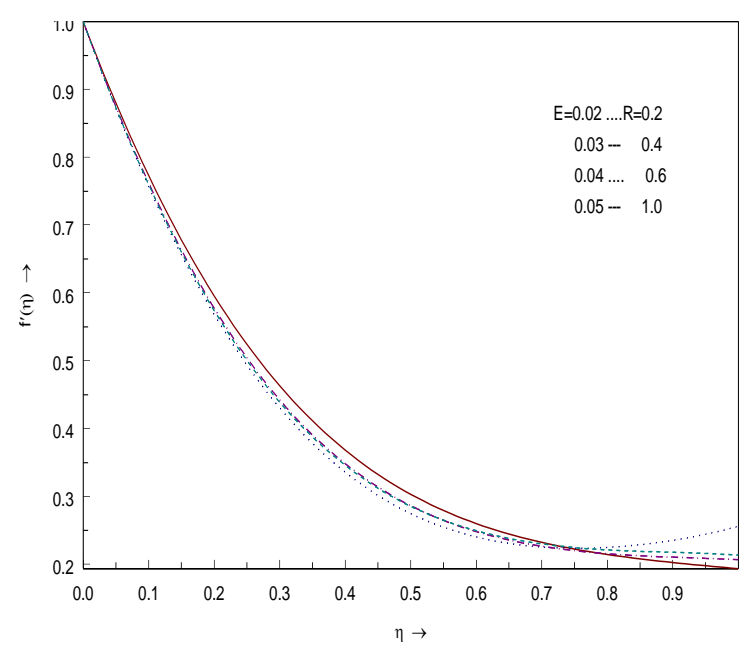

Fig.4 Effect of radiation parameter for various values of Prandtl number in PST case with E=0.02, B=$0.05, \mathrm{~K} 1=0.1, \mathrm{~K} 2=0.2$ for velocity profile

Table I provides the values of skin friction coefficient for various values of $\mathrm{Pr}, \mathrm{k} 1, \mathrm{k} 2, \theta_{r}$ and $\mathrm{R}$. The increases individual values of $\mathrm{Pr}, \mathrm{k} 1, \mathrm{k} 2, \theta_{r}$ and $\mathrm{R}$ are to decreases, the rate of heat transfer in skin friction coefficients. 


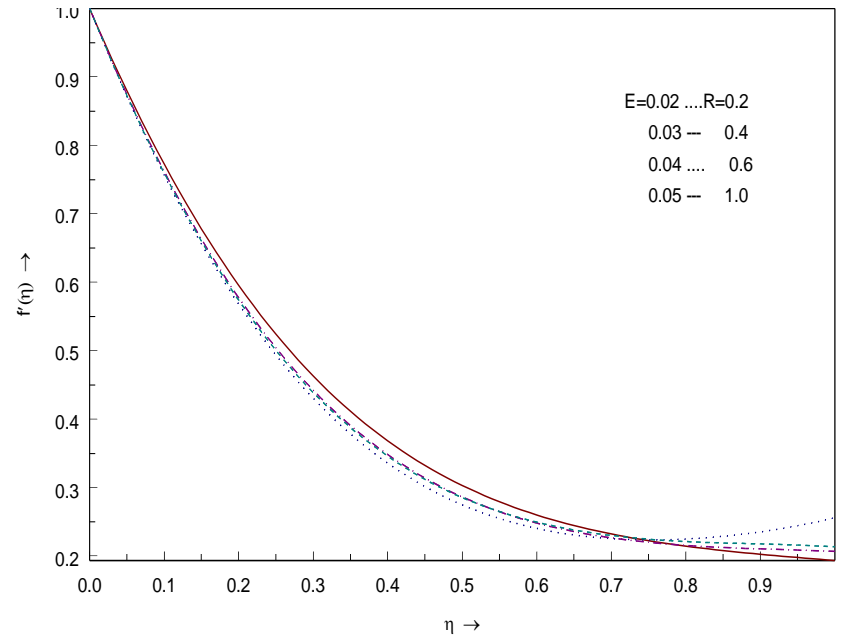

Fig.5 Effect of radiation parameter for various values of Eckert number in PST case with $\operatorname{Pr}=7.0, \mathrm{~B}=-0.05$, $\mathrm{K} 1=0.1, \mathrm{~K} 2=0.2$ for velocity profile

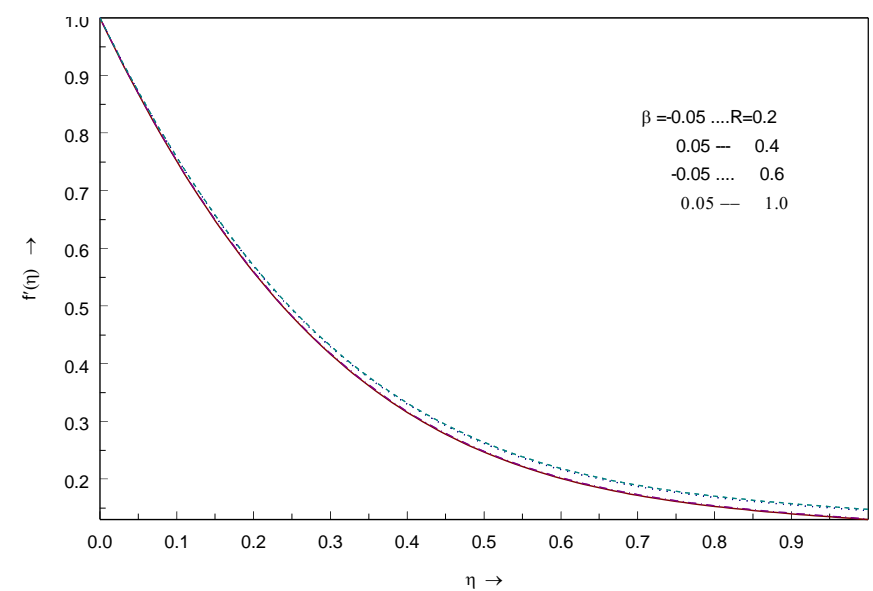

Fig 6 Effect of radiation parameter for various values of heat source/sink in PST case with $\operatorname{Pr}=7.0, \mathrm{E}=0.02$, $\mathrm{K} 1=0.1, \mathrm{~K} 2=0.2$ for velocity profile

Table I:Variation of $\mathrm{f}$ '(0) for different values of $\mathrm{Pr}, \mathrm{K} 1, \mathrm{k} 2, \mathrm{R}$ and A (PHF case)

\begin{tabular}{|c|c|c|c|c|c|c|}
\hline $\begin{array}{l}\text { Prandtl } \\
\text { number Pr }\end{array}$ & $\begin{array}{l}\text { Visco-elastic } \\
\text { parameter } \\
\text { k1 }\end{array}$ & $\begin{array}{l}\text { Porosity } \\
\text { Parameter } \\
\text { k2 }\end{array}$ & $\begin{array}{l}\text { Fluid Viscosity } \\
\text { Parameter } \\
\text { (A) }\end{array}$ & $\begin{array}{l}\text { Radiation } \\
\text { Parameter } \\
\text { (R) }\end{array}$ & $\begin{array}{l}\text { Present } \\
\text { result } \\
\text { (PST) }\end{array}$ & $\begin{array}{l}\text { Present } \\
\text { result } \\
\text { (PHF) }\end{array}$ \\
\hline \multirow[t]{4}{*}{7.0} & 0.1 & 0.0 & $\begin{array}{l}-1.0 \\
-10.0 \\
-1.0 \\
-10.0\end{array}$ & $\begin{array}{l}0.2 \\
0.4 \\
0.6 \\
1.0\end{array}$ & $\begin{array}{l}-1.803727 \\
-1.814058 \\
-1.800378 \\
-1.794640\end{array}$ & $\begin{array}{l}-1.794640 \\
-1.764740 \\
-1.664788 \\
-1.217190\end{array}$ \\
\hline & & 1.0 & $\begin{array}{l}-1.0 \\
-10.0 \\
-1.0 \\
-10.0\end{array}$ & $\begin{array}{l}0.2 \\
0.4 \\
0.6 \\
1.0\end{array}$ & $\begin{array}{l}-2.766242 \\
-2.758705 \\
-2.753568 \\
-2.755034\end{array}$ & $\begin{array}{l}-0.154601 \\
-0.159342 \\
-0.153365 \\
-0.154730\end{array}$ \\
\hline & 0.2 & $\mathbf{0 . 0}$ & $\begin{array}{l}-1.0 \\
-10.0 \\
-1.0 \\
-10.0\end{array}$ & $\begin{array}{l}0.2 \\
0.4 \\
0.6 \\
1.0\end{array}$ & $\begin{array}{l}-1.803521 \\
-1.824108 \\
-1.800178 \\
-1.784141\end{array}$ & $\begin{array}{l}-0.252098 \\
-0.249914 \\
-0.250445 \\
-0.249657\end{array}$ \\
\hline & & 1.0 & $\begin{array}{l}-1.0 \\
-10.0 \\
-1.0 \\
-10.0\end{array}$ & $\begin{array}{l}0.2 \\
0.4 \\
0.6 \\
1.0\end{array}$ & $\begin{array}{l}-2.771242 \\
-2.701142 \\
-2.701243 \\
-2.701122\end{array}$ & $\begin{array}{r}-0.219494 \\
-0.218273 \\
-0.218273 \\
-0.219698\end{array}$ \\
\hline
\end{tabular}




\section{Conclusion:}

The effect of heat radiation on the flow of walter's liquid B over a impermeable stretching sheet with heat source/sink and elastic parameter have been discussed. The analysis were carried out for two types of different heating process namely (i) Presribed surface temperature(PST) and (ii) Prescribed wall heat flux (PHF). The effects of emerging parameters have been and discussed through table in (PHF).

The conclusion derived from this study as mentioned below

1. It is found that the temperature profile and velocity profile decreases with the increasing value of the viscoelastic parameter, Eckert number and heat source/sink parameter.

2. The temperature decreases with the increasing value of Prandtl number, fluid viscosity parameter and radiation parameter.

3. The combined effect of increasing of radiation parameter with increasing visco elastic parameter, Eckert number, Prandtl number and fluid viscosity decreases the temperature profile and velocity profile.

\section{References}

[1]. Andersson,H.I., MHD flow of a visco-elastic fluid past a stretching surface, Acta. Mech., 95, 1992, 227-232

[2]. Abo-Eldahab Emad,M and EI AZIZ mohammed,A., Blowing suction effect on hydromagentic heat transfer by mixed convection from an inclined continuously stretching surface with internal heat generation/absorption, Int. J. Therm science, 45, 2004, 709-719

[3]. Bataller, P ., Effects of heat source/sink, radiation and work done by deformation on flow and heat transfer of a viscoelastic fluid over a stretching sheet, computer and maths with Appl., 35, 2007, 305-316

[4]. Chen, C.K and Char, M.I ., Heat transfer on a continuous stretching surfaces with suction or blowing, J. Math .Anal. Appl., 33, $1988,568-580$

[5]. Carragher, P and Crane L.J ., Heat transfer on a continuous stretching sheet, ZAM P, 12, 1982, 564-565

[6]. Dual pal, E and Hiranmoy Mondal,M ., Effects of soret Duffour, chemical reaction and thermal radiation on MHD non-Darcy stretching sheet, Int. J. Nonlinear Mech., 45, 2011, 1942-1958

[7]. Gupta, P.S and Gupta, A.S., Heat and mass transfer in a stretching sheet with suction or blowing, Can .J. Chem. Eng, 21, 1977, 744-746

[8]. Hayat, P ., Effects of radiation and magnetic field on the mixed convection stagnation point flow over a vertical stretching sheet in a porous medium, Int. J. Heat Mass Transfer., 30, 2010, 466-474

[9]. Lai, F.C and Kulacki, F.A., The effect of variable viscosity on convective heat transfer along a vertical surface in a saturated porous medium, Int.J. Heat Mass Transfer, 56, 1990, 10-28

[10]. Mahantesh, M and Vajravelu, K ., Heat transfer in MHD viscoelastic boundary layer flow over a stretching sheet with thermal radiation and non uniform heat source/sink, Int. J. Nonlinear mech., 24, 2011, 578-3590

[11]. Mohamed, E.M and Ouaf., Exact solution of thermal radiation on MHD flow over a stretching porous sheet, App.math. comp, 2005, 117-1125

[12]. Mohamed, E.M., Radiation effect on the flow and heat transfer over an unsteady stretching sheet, Chem Eng Sci, 89, 2009, 521-524

[13]. Prasad, K.V, Subhas Abel and Joshi, A., Oscillatory motion of a visco elastic fluid over a stretching sheet in porous media, 81, 2000, 61-68

[14]. Rajagopal, K.R, Na, T.Y and Gupat, A.S ., A non-similar boundary layer on a stretching sheet in a non-Newtonian fluid with uniform free stream, J. Math. Phys. Sci, 3. 1987, 187-200

[15]. Rafael Cortell, P., Effects of viscous dissipation and radiation on the thermal boundary layer over a nonlinearly stretching sheet, Int. J. Nonlinear Mech, 76, 2008, 631- 636

[16]. Raptis, A and Perdikis, C., Viscoelastic flow by the presence of radiation, Journal of Applied Mathematics and Physics(ZAMP), 30, 1998, 277-279

[17]. Subhas Abel, P.H. and Veen., Visco-elastic fluid flow and heat transfer in a porous medium over a stretching sheet, Lnt. J. NonLinear Mech, 113, 1998, 531-538

[18]. Siddheshwar and Mahabalaswar., Effects of radiation and heat source on MHD flow of a viscoelastic liquid and heat transfer over a stretching sheet, Int. J. Nonlinear Mech, 116, 2005, 807-820

[19]. Sakiadis, B.C., Bondary layer behavior on continuous solid surfaces: (Boundary layer equation for the two dimensional and axisymmetric flow, AIche, 7, 1961, 221-225

[20]. Sam Lawrence, P and Nageswara Rao, B., The non uniqueness of the MHD flow of a visco-elastic fluid past a stretching sheet, Acta Mech, 112, 1995, 223-228

[21]. Salem, A.M., Variable viscosity and thermal conductivity effects on MHD flow and heat transfer in viscoelastic fluid over a stretching sheet, Physics Letters A, 116, 2007, 315-322 